\title{
Scenario-based Economic Dispatch with Tunable Risk Levels in High-renewable Power Systems
}

\author{
M. Sadegh Modarresi, Student Member, IEEE, Le Xie, Senior Member, IEEE, Marco C. Campi, Fellow, IEEE, \\ Simone Garatti, Member, IEEE, Algo Carè, Anupam A. Thatte, Member, IEEE, and P. R. Kumar, Fellow, IEEE
}

\begin{abstract}
This paper introduces an empirical approach to dispatch resources in real-time power system operation with growing levels of uncertainties emerging from intermittent and distributed energy resources in the supply and the demand side. It is shown that by taking empirical data of specific sizes, the dispatch results can lead to a quantifiable and rigorous bound on the risk of violating constraints at the implementation stage. In particular, we formulate the look-ahead real-time economic dispatch problem using the scenario approach. This approach takes empirical data as input and guarantees a tunable probability of violating the constraints according to the input data size. By exploiting the structure of the economic dispatch, we show that in the absence of transmission constraints, the number of samples that is required by the theory does not grow with the size of the problem. In the more general case with transmission constraints, it is shown that the posterior bound on the risk of dispatch can be quantified and can be much smaller than the risk bound before solving the dispatch. Numerical examples based on a standard test system suggest that the scenario approach can provide a practically attractive solution with theoretically rigorous properties for risk-limiting power system operations.
\end{abstract}

Index Terms-Chance constrained programming, economic dispatch, electricity market, renewable generation, robust optimization, scenario approach.

\section{INTRODUCTION}

Increasing levels of uncertain distributed generation resources are being integrated into electric power systems. These new resources of energy are distributed both in the supply and the demand side. Wind generation and utility-scale solar farms are two examples of these resources on the supply side, and roof-top solar PVs is an example of these resources on the demand side. For instance, wind power currently comprises $22 \%$ of total generation capacity in Electric Reliability Council of Texas (ERCOT) and the record of the wind share in the total energy production in any one hour reached $54 \%$ on October 27, 2017 [1]. ERCOT also plans to integrate $8.3-11.9 \%$ of solar generation and retire a substantial portion of its coal generation resources over the next decade [2]. Similar trends are also

This work is supported in part by NSF Contracts ECCS-1760554 and ECCS-1839616, NSF Science \& Technology Center Grant CCF-0939370, and the Power Systems Engineering Research Center (PSERC).

M. S. Modarresi, L. Xie and P. R. Kumar are with the Department of Electrical and Computer Engineering, Texas A\&M University, College Station, TX, USA. Correspondence Author: Le Xie.

M. C. Campi and A. Carè are with Department of Information Engineering, University of Brescia, 25123 Brescia, Italy.

S. Garatti is with Dipartimento di Elettronica Informazione e Bioingegneria, Politecnico di Milano, Piazza Leonardo da Vinci 32, 20133 Milano, Italy.

A. A. Thatte is with the Midcontinent Independent System Operator, Carmel, IN 46032 USA.
TABLE I: Nomenclature
Sets:
$\Delta \quad$ The uncertainty set.
$\Delta_{\mathcal{S}} \quad$ Set of $\mathcal{S}$ scenarios which are randomly ex- tracted from uncertainty set $\Delta, \delta_{s} \in \Delta_{\mathcal{S}}$.
$\mathcal{A} \quad$ Set of scenarios to be eliminated by any arbitrary rule.
$c_{g_{i}} \quad$ Submitted energy offer curve of unit $g_{i}$.
$G, G^{r} \quad$ Set of operating generators, set of renewable generation resources, $G^{r} \subset G$.
$\mathcal{X} \quad$ Set of feasible solutions for the scenario problem (convex and closed), $x \in \mathcal{X}$.

Parameters and constants:

$\epsilon, \epsilon_{k} \quad$ Risk parameter, risk parameter after discarding $k$ scenarios.

$\bar{\epsilon} \quad$ Upper bound of the risk parameter in the aposteriori stage.

$\nu_{\mathcal{S}}^{\star} \quad$ Number of support constraints.

$\beta \quad$ Confidence parameter.

$B_{\mathbf{g}} \quad$ Nodal generation incident matrix.

$B_{1} \quad$ Nodal load incident matrix.

$d \quad$ Number of decision variables in the scenario problem $\mathcal{X} \subset R^{d}$.

$F, \bar{F} \quad$ Branches flow vector, branches capacity vector.

$g_{i}, l_{j} \quad$ Symbols of generator $g_{i}$ and load point $l_{j}$.

$N_{b}, N_{g}$

$N_{l}, N_{k} \quad$ Number of buses, generators, loads and lines.

$P T D F_{\mathbf{e}} \quad$ Extended power transfer distribution factor matrix.

$p_{\mathbf{g}} \quad$ Generation forecast error matrix, $\left(p_{g_{i}}=\right.$ $0 \quad \forall g_{i} \notin G^{r}$ ).

$\begin{array}{ll}p_{\mathbf{l}} & \text { Load forecast error matrix. } \\ \mathcal{P}_{\mathbf{n}} & \text { Nodal power injection matrix. }\end{array}$

$\hat{\mathcal{P}}_{1} \quad$ Load forecast matrix.

$R U_{g_{i}} \quad$ Upward ramp rate capacity of unit $g_{i}$.

$R D_{g_{i}} \quad$ Downward ramp rate capacity of unit $g_{i}$.

$T \quad$ Number of intervals of the LAED.

$z \quad$ Objective function.

Decision variables

$\mathcal{P}_{\mathrm{g}} \quad$ Power generation matrix.

$P_{g_{i}} \quad$ Power generation for $g_{i}$.

occurring elsewhere in the world. Operational planning such 
as unit commitment and dispatch will need to be revisited in order to reliably absorb these new resources at an affordable cost.

Due to the uncertainty and variability introduced by renewables, there has been a large body of literature devoted to solving Optimal Power Flow (OPF) [3], [4]. At the dayahead stage, there has been a large body of literature applying stochastic [5], [6] and robust [7], [8] optimization techniques in unit commitment problems, which involve integer variables. At the near real-time stage, there have been similar efforts on improving the performance of economic dispatch, or the optimal power flow problems. Among these efforts is lookahead economic dispatch (LAED), which employs a moving window optimization to account for inter-temporal variations in the near term [9]-[12]. The key idea is to extend the optimization horizon from one-time interval to multiple timecoupled intervals, allowing for early detection and better management of ramping/congestion related variations. However, how to model uncertainty for such a problem is still under exploration [13]-[18].

One can categorize the methods dealing with uncertainty into two general approaches. The first class of methods, which are categorized as robust optimization [19]-[21] try to address any realization of uncertainty. However, robust optimization guarantees feasibility only if realizations of uncertainty occur inside the predefined uncertainty set or dynamically evolving uncertainty set, and, second, the extent of introduced conservativeness is generally unknown [22].

The second approach consists in finding a solution that satisfies the constraints with a predefined (usually high) probability - chance-constrained programming (CCP). CCP often offers a trade-off between the level of conservativeness of the results and feasibility of the problem. CCP is NP-hard in general. Methods to deal with CCP include: finding a deterministic equivalent to the chance-constrained program [23], Big-M approach [24], [25], robust counterpart [26], [27], and the scenario approach [28], [29].

With the proliferation of sensors and computational power, it is becoming increasingly desirable to obtain insights from empirical data and observations. Scenario approach as a sample-based optimization techniques has several features that makes it desirable for operational decision making: (1) it is driven purely by samples of empirical data; (2) it provides theoretical guarantees on the risk of violating the constraints; (3) it might be able to present a much tighter upper bound on the risk after observing complexity of the solution, and (4) it provides the option of dropping some realizations of uncertainty or relaxing the constraints while keeping violation probabilities within defined bounds.

The scenario approach deals with high levels of uncertain resources and provides quantifiable risk levels at the implementation stage. Much of the uncertainties arise from the high penetration of distributed energy resources (DERs). There have been a number of efforts to exploit the scenario approach theory in the general field of optimization, control [30], [31], and power system adequacy and security assessment [32]-[35]. This work is an effort to exploit the potential of the scenario approach theory for real-time scheduling and dispatch with high level of renewable resource penetration. In particular, the focus of the paper is on the scalability of the scenario theory to the real-time power system operation which will be discussed in great details in Section III.

The theoretical guarantees provided by the scenario approach can be a-priori (before collecting data) or a-posteriori (after computing a solution $x^{\star}$ based on the collected data). Correspondingly, depending on the chosen type of guarantee, the implementation of the scenario approach can be different.

Specifically, according to the a-priori results, it is possible to determine the number of scenarios that are required to attain a specified level of risk with high confidence. In this case, the scenario algorithm consists of the following steps: Step 1, specify the risk tolerance and the level of confidence in the results; Step 2, acquire an adequate dataset of a size that guarantees the risk and confidence levels; Step 3: Solve the problem (finding $x^{\star}$ ) taking only the sampled scenarios into account; and Step 4 (optional), eliminate a subset of scenarios from the sample set, using any rule, resulting in a new quantifiable risk parameter, but with a solution $x^{\star \prime}$ with lower cost, $z\left(x^{\star \prime}\right) \leq z\left(x^{\star}\right)$ (trading risk for performance).

Along the a-posteriori approach, instead, the scenario algorithm is run with a given dataset of any size. Then, after computing the solution, one can analyze its complexity (defined precisely in Section III), and, by studying the risk jointly with the complexity, one can obtain a more clear knowledge of the actual risk of the scenario solution. This process is called Wait-and-Judge approach in [36]. It will be shown that this can play a key role vis-a-vis the scalability of the scenario approach method for bulk power systems applications.

The main contributions of this paper are as follows:

- A scenario approach-based formulation of LAED (ScLAED) that provides a guarantee on the risk level for any underlying distribution of uncertainty in the generation and/or demand is proposed.

- Conditions on the size of the dataset are derived under which the risk does not exceed a certain threshold with high probability.

- To address scalability of scenario approach theory to the real time power system operation, it is shown that there are cases where the size of the dataset needed to guarantee a risk threshold is independent of the size of LAED (i.e. the number of generators). Moreover in general, it is shown that the risk guarantees can be tightened to a more precise upper bound a-posteriori by observing the complexity of the Sc-LAED solution.

- Scenario reduction in Sc-LAED is also considered: one can use any rule to eliminate scenarios and thereby avoid overly conservative solutions, with measurable risk parameter changes.

The rest of this paper is organized as follows. Section II presents the formulation of the LAED. Section III presents the methods and key theoretical results of the scenario-based dispatch. Section IV presents case studies using a 2000-bus test case. Conclusions and future work are presented in Section V. 


\section{TAXONOMY OF LOOK-AHEAD ECONOMIC DISPATCH UNDER UNCERTAINTY}

A comparison between different LAED problem formulations is presented in this section. State of the art approaches in solving LAED are briefly discussed along with the proposed formulation for the Sc-LAED. The results in the first decision interval, e.g., $t=1$ are binding and the decisions for future intervals are considered as advisory and subject to change during future dispatch intervals.

\section{A. Deterministic, stochastic and robust LAED}

The most common approach to look-ahead economic dispatch is a deterministic one (1). The dispatch aims at balancing the deterministic forecasted demand with least cost while satisfying the constraints. This type of dispatch uses the least number of decision variables and/or constraints, and it is naturally formulated as a linear programming problem. Therefore it is easier to adopt in real-time market clearing processes. However, it was not designed to take decisions against forecast uncertainty:

$$
\begin{array}{ll}
\min _{P_{g_{i}}[t]} & z=\sum_{t=1}^{T} \sum_{i=1}^{N_{g}} c_{g_{i}}[t] P_{g_{i}}[t] \\
\text { s.t. } \quad & \sum_{i=1}^{N_{g}} P_{g_{i}}[t]=\sum_{j=1}^{N_{l}} \hat{P}_{l_{j}}[t] \quad \forall t=1,2, \ldots T \\
& -\bar{F} \leq F[t] \leq \bar{F}, \quad \forall t=1,2, \ldots T \\
& R D_{g_{i}} \leq P_{g_{i}}[t]-P_{g_{i}}[t-1] \leq R U_{g_{i}} \\
& \forall t=1,2, \ldots T, \forall i, \\
& P_{g_{i}}^{\min }[t] \leq P_{g_{i}}[t] \leq P_{g_{i}}^{\max }[t] \quad \forall t=1,2, \ldots T, \forall i .
\end{array}
$$

Supply and demand balance is enforced by (1b). Inequalities (1c), (1d), and (1e) are transmission flow limits, generator ramp-up and ramp-down constraints, and offered minimum and maximum generator capacities respectively for each interval. Line flow limits are modeled as element-wise inequalities in (1c), where $F=P T D F_{\mathbf{e}} \times \mathcal{P}_{\mathbf{n}} . P T D F_{\mathbf{e}}$ is the extended power transfer distribution factor matrix with a zero column vector inserted on the slack bus. Therefore the size of this matrix at each time $t$ is $N_{k} \times N_{b}$ and $\mathcal{P}_{\mathbf{n}}$ is the nodal power injection matrix [37]. $\mathcal{P}_{\mathbf{n}}$ can be calculated as (2). The forecast errors for generation and load are not being considered in the deterministic formulation and will be discussed in Subsection II-B. Elements of incident matrices, $B^{\mathrm{g}}$ and $B^{\mathrm{l}}$, for each node $n$ are as (3) and (4).

$$
\begin{aligned}
& \mathcal{P}_{\mathbf{n}}=B_{\mathbf{g}}\left(P_{\mathbf{g}}+p_{\mathbf{g}}\right)-B_{\mathbf{l}}\left(\hat{P}_{\mathbf{l}}+p_{\mathbf{l}}\right) \\
& b_{n, g_{i}}^{\mathbf{g}}=\left\{\begin{array}{cc}
1 & \text { if } g_{i} \underset{\text { bus }}{\stackrel{\text { connected } \text { to }}{\longrightarrow}} n \\
0 & \text { otherwise }
\end{array}\right\}, \\
& b_{n, l_{j}}^{\mathbf{l}}=\left\{\begin{array}{cc}
1 & \text { if } l_{j} \underset{\text { bus }}{\stackrel{\text { connected } \text { to }}{\longrightarrow}} n \\
0 & \text { otherwise }
\end{array}\right\} \text {. }
\end{aligned}
$$

In contrast to the deterministic approach, stochastic and robust LAED consider the uncertainty for the non-binding time intervals. Stochastic LAED minimizes the overall expected cost of dispatch by incorporating the probability distribution of the forecast error.

In robust LAED, an optimal solution is sought that is feasible for all of the realizations of uncertainty in the system. Therefore, if robust LAED finds a feasible solution, then the problem is indeed feasible provided that the uncertainty set considered in the model accurately reflects the underlying uncertainty. In contrast to the stochastic approach, robust LAED requires less information about the underlying uncertainty, but can return conservative results. The robust LAED formulation that we follow in this paper is based upon [17].

\section{B. Scenario approach LAED}

Sc-LAED is defined as in (5), where $\mathcal{S}$ scenarios, $\delta_{1}, \ldots, \delta_{\mathcal{S}}$, are extracted from an uncertainty set $\Delta$ according to a probability distribution $\mathbb{P}$, and are simultaneously enforced [28], [38]. The main difference between the Sc-LAED formulation and robust LAED is in their approach toward uncertainty. While some robust LAED methods confine the borders of the uncertainty set (e.g., at $\mu \pm 3 \sigma$ ), [17], [39], this choice impacts on the robustness and the conservatism of the results in a way that is difficult to quantify and deal with. On the other hand, the scenario approach is a direct approach, that is, data (the sampled scenarios) are used directly in (5), without any preliminary design of the uncertainty set. Thus, in ScLAED, the set of the uncertain values for which the constraints are enforced is the set of the sampled scenarios, and, as we shall see, there are theorems that show how the number of sampled scenarios can be used to tune the probability that the obtained solution is satisfied by the unobserved uncertain values.

A crucial fact is that knowledge of the probability measure $\mathbb{P}$ over scenarios is not required: all that is required is a historical set of scenarios from the past that have already occurred. This sets the scenario approach apart from classic stochastic approaches where $\mathbb{P}$ is assumed to be known.

Note also that it is often the case that $\mathbb{P}$ is only implicitly defined by a complex model of the reality. In this case, valid scenarios (i.e., scenarios that are independent and identically distributed (i.i.d.)) can be generated by simulations, e.g. by resorting to statistical weather models [40]. The key theoretical guarantee provided by the scenario approach is that there is a rigorous upper bound on the level of risk associated with this type of dispatch, along with specified confidence level. For more discussion on the fundamental differences between the scenario approach and other approaches the reader is referred 
to [41].

$$
\begin{array}{ll}
\min _{P_{g_{i}}[t]} & z=\sum_{t=1}^{T} \sum_{i=1}^{N_{g}} c_{g_{i}}[t] P_{g_{i}}[t] \\
\text { s.t. } \quad & \sum_{i=1}^{N_{g}} P_{g_{i}}[t]=\sum_{j=1}^{N_{l}} \hat{P}_{l_{j}}[t], \quad t=1 \\
& \sum_{i=1}^{N_{g}} P_{g_{i}}[t] \geq \sum_{j=1}^{N_{l}} \hat{P}_{l_{j}}[t]+p^{\delta_{s}}[t] \\
& \forall \delta_{s} \in \Delta_{\mathcal{S}}, \forall t=2,3, \ldots T \\
& -\bar{F} \leq F^{\delta_{s}}[t] \leq \bar{F}, \quad \forall \delta_{s} \in \Delta_{\mathcal{S}}, \forall t=1,2, \ldots T \\
& R D_{g_{i}} \leq P_{g_{i}}[t]-P_{g_{i}}[t-1] \leq R U_{g_{i}} \\
& \forall t=1,2, \ldots T, \forall i, \\
& P_{g_{i}}^{\min }[t] \leq P_{g_{i}}[t] \leq P_{g_{i}}^{\max }[t] \quad \forall t=1,2, \ldots T, \forall i
\end{array}
$$

We now consider (5) in more details. The role of constraints are the same as that discussed for (1), with two differences. First the power balancing constraint is modeled as an equality constraint for the binding (and deterministic) interval, whereas it is modeled as an inequality for the nonbinding (and subject to the uncertainty) interval. The intent of modeling (5c) as inequality is to have enough capacity to respond to all unexpected changes in the load and generation [17]. Second, (5c) and (5d) are scenario-dependent constraints. $p^{\delta_{s}}[t]$ in (5c) is the net forecast error of the load and intermittent energy resources under scenario $\delta_{s}$ and is defined as: $p^{\delta_{s}}[t]=\sum_{j=1}^{N_{l}} p_{l_{j}}^{\delta_{s}}[t]-\sum_{i=1}^{N_{g}} p_{g_{i}}^{\delta_{s}}[t], \quad p_{g_{i}}=0 \quad \forall g_{i} \notin G^{r}$. For resources with time-varying upper bound on generation, such as wind and solar, $P_{g_{i}}^{\max }[t]$ is the maximum sustained limit of generation submitted by the resource at time $t$.

For simplicity, we rewrite (5) as (6), where (6b) represents $(5 \mathrm{~b}, 5 \mathrm{e}, 5 \mathrm{f})$ and $(6 \mathrm{c})$ represents $(5 \mathrm{c}, 5 \mathrm{~d})$ :

$$
\begin{array}{ll}
\min _{x \in \mathcal{X}} & z=c^{T} x \\
\text { s.t. } & f_{1}(x) \leq 0, \\
& f_{2}\left(x, \delta_{s}\right) \leq 0, \quad \forall \delta_{s} \in \Delta_{\mathcal{S}} .
\end{array}
$$

As we shall see, the number $\mathcal{S}$ of scenarios that guarantees a certain risk level can be computed independently of the underlying probability distribution of uncertainties, and mainly depends on the level of risk that one is willing to tolerate and the number of decision variables. If some scenarios cause unacceptable increments in cost (or result in infeasibility visa-vis dispatch), they can be removed from $\Delta_{\mathcal{S}}$ with controlled and quantifiable increase on the risk parameter, $\epsilon$. Also, after finding a solution to (5), it can be shown that one can have access to the upper bound on conservativeness of the resulting solution. Further details on exploiting the scenario approach theory for the purpose of solving (5) are discussed in Section III.

Before a deeper investigation of the scenario theory, a brief discussion about the impact of Sc-LAED on electricity market nodal pricing might be desirable. Locational Marginal Pricing is the main approach to define electricity prices and transmission congestion costs in many wholesale markets. To calculate the Locational Marginal Prices (LMPs) in Sc-LAED, a process similar to ex-post LMP calculation known as the pricing run in Independent System Operators (ISO) such as PJM, ISO New England, and NYISO [42] should occur. The inputs to the LMP calculation are the results from the ScLAED binding interval and the subset of active transmission constraints. Essentially the results for $t=1$ from (5) are used to find LMPs by solving a single interval problem. There are different approaches to calculate the ex-post LMPs such as [42]-[44]. The Appendix describes one of the most common approaches based on [45], [46] for the structure of our problem as (5).

Sc-LAED does not have a direct impact on markets clearing before the real-time market. For instance, Financial Transmission Rights (FTRs) allow market participants to hedge against transmission congestion charges present in the dayahead market [47]. However, Sc-LAED might have an impact on market participants profiting from the differences between real-time and day-ahead prices. For instance, for the case of virtual transactions. The value of these products depends on the differences between day-ahead and real-time prices. Without a detailed simulation study, it is difficult to comment on the impact of Sc-LAED compared to conventional dispatch in terms of impact on Virtual trading profit/loss.

\section{Computational Algorithm to Solve the SCENARIO APPROACH ECONOMIC DISPATCH}

The Sc-LAED approach prescribes to solve a convex optimization problem whose constraints depend on sampled scenarios, which are expected to carry knowledge about the future behavior of load, wind and solar resources. Precisely, a set of scenarios $\Delta_{\mathcal{S}} \subset \Delta$ is obtained by sampling the uncertainty set $\Delta$, and the problem (6) is then solved for the scenarios belonging to $\Delta_{\mathcal{S}}$. Problem (6) is an instance of what in the literature is called a Scenario Problem (or Scenario Program, see e.g., [28], [29], [36]), and we will denote it by $S P_{\mathcal{S}}$.

The central question that arises is therefore the following: how much one can rely on $\Delta_{\mathcal{S}}$ as a representative of the whole uncertainty set $\Delta$, which includes all the possible (yet unseen) realizations of the stochastic uncertainty? In order to address this question in quantitative terms, we have to define rigorously the risk of the solution $x_{\mathcal{S}}^{\star}$, as the probability that this solution will turn out to be infeasible for another realization of the stochastic uncertainty. This concept is made precise by defining the violation probability of $x_{\mathcal{S}}^{\star}$ as follows.

Definition 1 (Violation probability (or risk) of $x_{\mathcal{S}}^{\star}$ ): Let $x_{\mathcal{S}}^{\star} \in$ $\mathcal{X}_{\mathcal{S}}$ be the solution to $S P_{\mathcal{S}}$. Then the violation probability (or risk) of $x_{\mathcal{S}}^{\star}$ is denoted by $V\left(x_{\mathcal{S}}^{\star}\right)$ and defined as

$$
V\left(x_{\mathcal{S}}^{\star}\right):=\mathbb{P}\left\{\delta \in \Delta: f_{2}\left(x^{\star}, \delta\right)>0\right\},
$$

where we recall that $\mathbb{P}$ is the probability distribution over $\Delta$ according to which the scenarios are sampled in an independent and identically distributed way.

Clearly, the risk depends on the set of extracted scenarios, and therefore it has a stochastic variability. Nevertheless, in 
[28] it was proven that there are conditions under which the risk is distributed according to a beta distribution, irrespective of the distribution of the sampling probability $\mathbb{P}$. More in general, the results in [28], and in following contributions, allow one to compute upper-bounds to the risk that hold true with high confidence. A crucial role in the theory of the scenario approach is played by the concept of support constraint, which is defined as follows.

Definition 2 (Support Constraint): The scenario-dependent constraint corresponding to sample $\delta_{s}, s \in\{1,2, \ldots, \mathcal{S}\}$, is a support constraint for $S P_{\mathcal{S}}$, if its removal improves the solution of $S P_{\mathcal{S}}$, i.e., if it decreases the optimal cost (6a).

We are now ready to state the main results of the theory of the scenario approach and exploit them in the present context. Subsections III-A and III-B focus on the a-priori evaluation of the risk, where we use samples from the uncertainty set to guarantee a certain level of risk with high confidence. Based on the results in these two subsections and the analysis of the Sc-LAED problem in the absence of congestion, we propose a data-driven procedure that we call Algorithm 1. In Subsection III-C, we consider the case when there is congestion and show that, in spite of the high number of scenarios that are required by the a-priori approach, it is still possible to make useful and accurate claims on the risk after observing the complexity of the obtained solution (a-posteriori evaluation). Conclusions are drawn and a data-driven procedure that exploits a-posteriori evaluation is proposed (Algorithm 2).

\section{A. The a-priori scenario approach method}

The main theorem in [28] is the following one.

Theorem 1: With the assumption that (6) returns a unique solution, it holds that

$$
\mathbb{P}^{\mathcal{S}}\left\{V\left(x_{\mathcal{S}}^{\star}\right)>\epsilon\right\} \leq \sum_{i=0}^{d-1}\left(\begin{array}{c}
\mathcal{S} \\
i
\end{array}\right) \epsilon^{i}(1-\epsilon)^{\mathcal{S}-i},
$$

where $\mathbb{P}^{\mathcal{S}}$ is the probability distribution taken over $\delta_{1}, \ldots, \delta_{\mathcal{S}}$, which is a product probability due to independence.

The right-hand side of (8) is the tail of a beta distribution with parameters $(d, \mathcal{S}-d+1)$. As $\mathcal{S}$ grows, the tail goes exponentially to zero [28]. Fixing a small $\beta$, say $\beta=10^{-6}$, one can easily find the smallest number of samples $\mathcal{S}$ such that $\sum_{i=0}^{d-1}\left(\begin{array}{c}\mathcal{S} \\ i\end{array}\right) \epsilon^{i}(1-\epsilon)^{\mathcal{S}-i}<\beta$ holds true, so that the right-hand side of (8) is less than the specified $\beta$. Then, one can claim that with high confidence $1-\beta$ the risk $V\left(x_{\mathcal{S}}^{\star}\right)$ of the scenario solution with $\mathcal{S}$ scenarios is no larger than $\epsilon$. Note that the right-hand side of (8) does not depend on $\mathbb{P}$. This is remarkable and shows that, in order to guarantee that $V\left(x_{\mathcal{S}}^{\star}\right) \leq \epsilon$ with confidence $1-\beta$, we do not need to know $\mathbb{P}$.

A graphical representation of the roles of the risk parameter $\epsilon$ and the confidence parameter $\beta$ is shown in Fig. 1. The cube on the left is $\Delta_{\mathcal{S}}$, the set of all the possible $\mathcal{S}$-tuples of scenarios. A point in this cube can be identified with an instance of $\Delta_{\mathcal{S}}$, i.e., with a particular set of scenarios $\left\{\delta_{1}, \delta_{2}, \ldots, \delta_{\mathcal{S}}\right\}$ that is obtained by randomly sampling $\mathcal{S}$ scenarios from $\Delta$ according to the probability distribution $\mathbb{P}$. For this sample $\Delta_{\mathcal{S}}$, there is a set of feasible solutions $\chi$ which does not violate any of the constraints for any of the

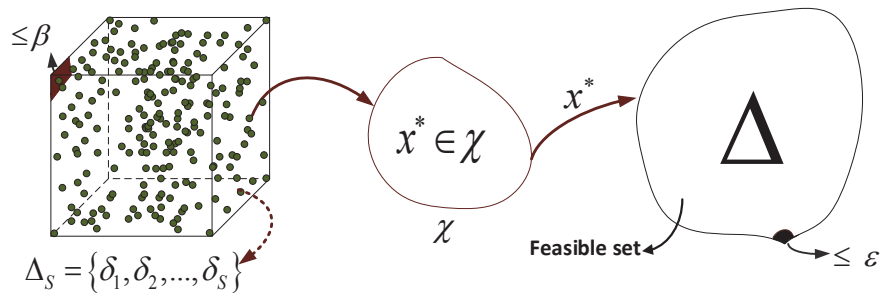

Fig. 1: Illustration of the scenario approach.

scenarios in $\Delta_{\mathcal{S}}$. This is depicted in the middle of Fig. 1. An optimal solution $x_{\mathcal{S}}^{\star}$ is then determined for this set $\Delta_{\mathcal{S}}$ of scenarios. The set of scenarios $\delta$ belonging to $\Delta$ for which $f_{2}\left(x_{\mathcal{S}}^{\star}, \delta\right)>0$ (i.e. the constraint in (6) is violated) is called the violation region and it is the region shaded black in the right in Fig. 1. This region has probability $V\left(x_{\mathcal{S}}^{\star}\right)$. We would like this probability to be always smaller than the risk parameter $\epsilon$. However, $V\left(x_{\mathcal{S}}^{\star}\right)$ has a variability as it depends on the sampled scenarios $\Delta_{\mathcal{S}}$ through $x_{\mathcal{S}}^{\star}$, and it will happen that $V\left(x_{\mathcal{S}}^{\star}\right)>\epsilon$ for certain samples $\Delta_{\mathcal{S}}$ that are in a bad set. Such bad set is depicted as the black region in the cube on the left. Theorem 1 guarantees that, if the right-hand side of (8) is smaller than $\beta \in(0,1)$, the bad set has a probability that is smaller than $\beta$ (with respect to the product measure $\mathbb{P}^{\mathcal{S}}$ ).

An explicit formula to find $\mathcal{S}$, which returns a slightly more conservative number of samples, is given below in (9), which is taken from [48]. As can be seen, the number of samples needed grows linearly with the dimension the optimization being performed and $\frac{1}{\epsilon}$, but it is not as sensitive to $\beta$.

Lemma 1: Under the same conditions as Theorem 1, if

$$
\mathcal{S} \geq \frac{2}{\epsilon}\left(\ln \frac{1}{\beta}+d\right)
$$

then $\mathbb{P}^{\mathcal{S}}\left\{V\left(x_{\mathcal{S}}^{\star}\right)>\epsilon\right\} \leq \beta$.

We now consider the structure of the Sc-LAED problem more explicitly. It is remarkable that in (5), only (5c) and (5d) consist of scenario dependent constraints defined by the netload forecast error at each bus. Eliminating (5d) (for now), one can observe that at most $T-1$ constraints can be active and indeed be support constraints. This is due to the fact that for each $t=2, \ldots, T$, the constraints in $(5 \mathrm{c})$ are half-spaces with the same slope but different displacement, so that no more than one can be active at the same time. Therefore, the number of support constraints for (5) is no more than $T-1$ with probability one. In view of this fact, the same formula in (8) can be applied by replacing $d$ with $T-1$, see e.g. [49], [50]. This prevents the number of samples from growing to very large numbers when congestion is not in the picture. The reduction in the number of required samples in this special case helps the scalability of the problem and shows that the number of samples can be independent of the number of generators and the number of buses in the system, and it only depends on the number of look-ahead intervals $T-1, \epsilon$ and $\beta$.

For a general case, and for bulk power systems application, satisfying (8) or (9) will require a large number of samples. This is an instance of a well known issue in the literature on the scenario approach, and several solutions are available 
that range from multiple steps or iterative procedures, see [51] and references therein, to regularization schemes, [52]. Among them, the recently proposed "wait and judge approach", [36], is of particular interest in the case of Sc-LAED, because it allows one to compute the upper bound on the risk of the solution as a function of the complexity of the obtained solution. In this way, useful upper bounds can be obtained also when a small amount of scenarios is available. This approach will be discussed in III-C.

It is also important to remark that, in general, among the sampled scenarios, there might be some extreme scenarios that can lead to excessively conservative results in terms of cost function. In the following subsection III-B, we show how to eliminate such scenarios while taking under control the increase in the risk bounds.

\section{B. Sampling and Discarding Approach in Sc-LAED}

The Sampling and Discarding Approach [29] is one technique in the scenario approach theory to trade risk for performance. Essentially the cost of Sc-LAED is reduced by eliminating scenarios of choice, but the price paid is an increase in the guaranteed risk. Let $\mathcal{A}$ be the discarded scenarios among those in $\Delta_{\mathcal{S}}$, and let $|\mathcal{A}|$ be the cardinality of $\mathcal{A}$. If the following relation is satisfied,

$$
\left(\begin{array}{c}
|\mathcal{A}|+d-1 \\
|\mathcal{A}|
\end{array}\right) \sum_{i=0}^{|\mathcal{A}|+d-1}\left(\begin{array}{c}
\mathcal{S} \\
i
\end{array}\right) \epsilon^{i}(1-\epsilon)^{\mathcal{S}-i} \leq \beta,
$$

then the solution $x_{\mathcal{S}-|\mathcal{A}|}^{\star}$ that is obtained by removing the scenarios in $\mathcal{A}$ from $\Delta_{\mathcal{S}}$ has a risk no larger than $\epsilon$, with high confidence $1-\beta$.

Usually, the support constraints with highest improvement in the cost of Sc-LAED are removed sequentially, by selecting the scenarios with the highest Lagrange multipliers. However, any other elimination rule is valid. For the stated result to hold true, the number of scenarios to be discarded $(|\mathcal{A}|)$ should be defined a-priori, while choosing $|\mathcal{A}|$ a-posteriori is possible at the price of a (usually minor) degradation in the overall confidence (typically, the confidence becomes $1-K \beta$ instead of $1-\beta$, where $K$ is the total number of values of $|\mathcal{A}|$ that one is willing to accept; for a detailed discussion on this point, see the discussion before equation (4) in [29]).

Combining the results of Theorem 1 and (10), a procedure (Algorithm 1) is here proposed for the case when no congestion is expected. The user inputs a desired risk parameter $\epsilon_{0}$. As explained above, exploring alternative solutions through scenario removal comes at the cost of degrading the guaranteed risk. Hence, the user also sets a modified risk parameter, $\tilde{\epsilon} \geq \epsilon_{0}$, which is still acceptable for practical purposes and that should be preferred to $\epsilon_{0}$ only if the gain in terms of cost function is significant. Similarly, a desired confidence parameter $\beta_{0}$ is specified together with a degraded confidence parameter $\tilde{\beta} \geq \beta_{0}$ that is still acceptable for practical purposes. These parameters together determine how many scenarios can be safely removed before a solution is returned by the algorithm, that is, they allow the system operator to trade risk for performance in a safe way.

Algorithm 1 for Sc-LAED in the absence of congestion

1) INPUT: $\epsilon_{0}, \tilde{\epsilon}, \beta_{0}, \tilde{\beta}, T$

2) Compute $\mathcal{S}$ that satisfies (9) when $\epsilon, \beta, d$ in (9) are replaced by $\epsilon_{0}, \beta_{0}, T-1$ respectively.

3) for $i=1,2, \ldots$

a) Find a valid $\epsilon_{i}$ that satisfies inequality (10) where $d$ and $|\mathcal{A}|$ in (10) are replaced by $T-1$ and $i$ respectively.

b) if $\left(\epsilon_{i}>\tilde{\epsilon}\right.$ or $\left.(i+1) \beta>\tilde{\beta}\right)$, then go to step 4 .

4) Sample $\mathcal{S}$ scenarios and compute $x_{\mathcal{S}}^{\star}$ by solving (6).

5) if $\left(c^{T} x_{\mathcal{S}}^{\star}\right.$ is satisfactory or $\left.i=1\right)$ then OUTPUT: $x_{\mathcal{S}}^{\star}$, its guaranteed risk $\epsilon_{0}$ and the confidence $\left(1-i \beta_{0}\right)$; else

6) for $k=1, \ldots, i-1$

a) Remove the worst $k$ scenarios from $\delta_{1}, \ldots, \delta_{\mathcal{S}}$ in (6) and compute the solution $x_{\mathcal{S}-k}^{\star}$ with $\mathcal{S}-k$ scenarios.

b) If ( $c^{T} x_{\mathcal{S}-k}^{\star}$ is satisfactory or $k$ is equal to $\left.i-1\right)$, then OUTPUT: $x_{\mathcal{S}-k}^{\star}$, its guaranteed risk $\epsilon_{k}$ and the confidence $\left(1-i \beta_{0}\right)$.

\section{The a-posteriori scenario approach method}

Convex optimization in dimension $d$ has, at most, $d$ support constraints [38], [53]. For the class of fully supported problems (when a problem in dimension $d$ has exactly $d$ support constraints with probability one), strict equality holds instead of inequality in (8). However, in many engineering applications, the problem being solved is not a fully supported problem. For instance, as discussed in Sc-LAED, when the system is not congested the number of support constraints is always far less than the number of decision variables. In this subsection, we study $V\left(x_{\mathcal{S}}\right)$ jointly with the complexity of the solution, defined below as $\nu_{\mathcal{S}}^{\star}$ for the general case where transmission constraints are considered.

Definition 3 (Complexity): $\nu_{\mathcal{S}}^{\star}$, the complexity of the solution $x_{\mathcal{S}}^{*}$ to $S P_{\mathcal{S}}$, is the number of the support constraints for $S P_{\mathcal{S}}$.

Complexity in Sc-LAED consists of the (at most $T-1$ ) support constraints corresponding to the generation adequacy constraint in (5c) plus possibly some support constraints for (5d), which cannot be predicted before solving (5).

The relation between risk and complexity was first studied in [36]. The results of [36] provide an upper bound on the risk after computing the solution. See Theorem 2 below.

Theorem 2: For program (6) with $\mathcal{S}>d$, for any $\tau=$ $0,1,2, \ldots, d$, the polynomial (11) below, with $t$ as variable has one and only one solution in $(0,1)$.

$$
\frac{\beta}{\mathcal{S}+1} \sum_{i=\tau}^{\mathcal{S}}\left(\begin{array}{l}
i \\
\tau
\end{array}\right) t^{i-\tau}-\left(\begin{array}{l}
\mathcal{S} \\
\tau
\end{array}\right) t^{\mathcal{S}-\tau}=0 .
$$

We denote this solution by $t(\tau)$. Defining $\bar{\epsilon}(\tau)=1-t(\tau)$ under the assumption of non-degeneracy and uniqueness of the solution [36], it holds that

$$
\mathbb{P}^{\mathcal{S}}\left\{V\left(x_{\mathcal{S}}^{\star}\right) \leq \bar{\epsilon}\left(\nu_{\mathcal{S}}^{\star}\right)\right\} \geq 1-\beta .
$$

The results after observing $\nu_{\mathcal{S}}^{\star}$ support constraints, compared to the original bound from [28] for the Synthetic Texas 


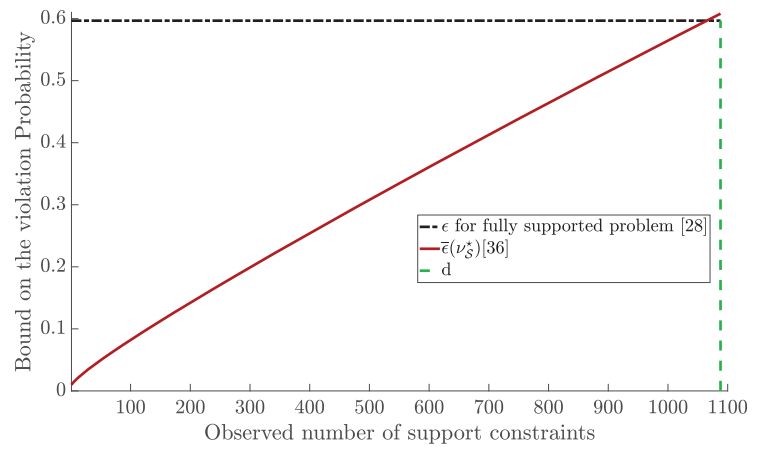

Fig. 2: Upper bound on the risk for $\mathcal{S}=2000, d=1088$. The vertical axis denotes values of $V\left(x_{\mathcal{S}}^{\star}\right)$, and horizontal axis denotes values of $\nu_{\mathcal{S}}^{\star}$. The distance between the black dotted line and the red curve is the improvement on the risk bounds provided by Theorem. 2 .

System [54] with $T=2$ in (5), are showed in Fig. 2. When $\nu_{\mathcal{S}}^{\star} \ll d$, the results improve significantly. This allows one to make significant claims on the risk even when the number of sampled scenarios is relatively small. For example, for the setting described in Fig. 2, an upper bound of $\epsilon=0.5967$ is obtained by using Theorem 1 with $\mathcal{S}=2000$. On the other hand, with the same number of scenarios, observing $\nu_{\mathcal{S}}^{\star}=18$ allows one to claim $\bar{\epsilon}\left(\nu_{\mathcal{S}}^{\star}\right)=0.0262$ as an upper bound thanks to Theorem 2.

The following Algorithm 2 exploits Theorem 2 to compute upper bounds on the risk of the scenario solution when congestion is expected, so that $d$ cannot be replaced by $T-1$ in Theorem 1, and the number of scenarios $\mathcal{S}$ cannot be increased to the values required by Theorem 1 . In this algorithm $\mathcal{S}$ is supposed to be given and typically it accounts for existing computational/data collection limitations.

Algorithm 2 for Sc-LAED when congestion is expected

1) INPUT: $\mathcal{S}, \beta$

2) Compute $\bar{\epsilon}(\tau), \tau=0, \ldots, d$ according to Theorem 2 .

3) Sample $\mathcal{S}$ scenarios and solve (6); obtain $x_{\mathcal{S}}^{\star}$ and count the number of support constraints $\nu_{\mathcal{S}}^{\star}$.

4) OUTPUT: $x_{\mathcal{S}}^{\star}$ and the upper bound on the risk $\bar{\epsilon}\left(\nu_{\mathcal{S}}^{\star}\right)$.

In conclusion, Algorithm 1 is the choice when the system operator does not expect congestion in the next $T$ intervals. On the other hand, when congestion is in the picture, the aposteriori approach (Algorithm 2) should be employed.

Considering that, in real life, the LAED problem is solved several times along a time horizon, one can try to guess $\nu_{\mathcal{S}}^{\star}$ for a new instance of Sc-LAED based on the past solutions, so as to adjust $\mathcal{S}$ accordingly. For example, $\nu_{\mathcal{S}}^{\star}[t-1]$, i.e., the number of support constraints at the previous time step, can be used as a starting estimate for the number of support constraints at time $t$. When $\nu_{\mathcal{S}}^{\star}[t-1] \ll \nu_{\mathcal{S}}^{\star}[t]$ and $\mathcal{S}$ samples are not sufficient to guarantee the desired risk level, one might sample new scenarios according to an iterative algorithm. Iterative schemes in this line of thought are the subject of ongoing research.

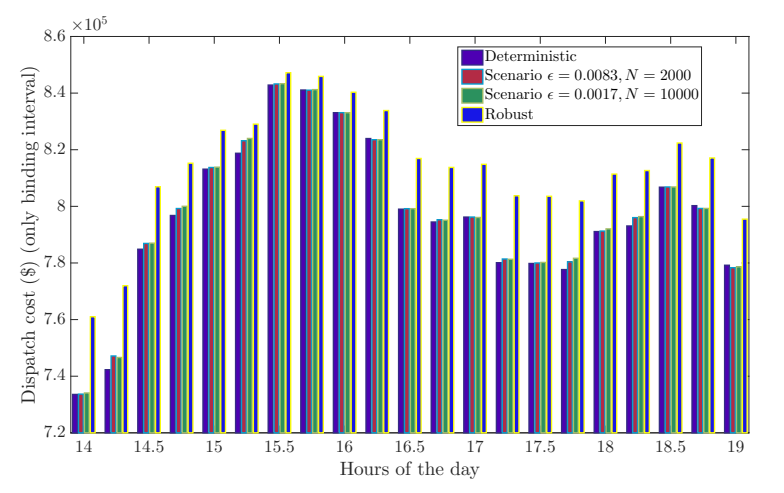

Fig. 3: Comparison of the Dispatch Cost during the peak hours of the day using different methods.

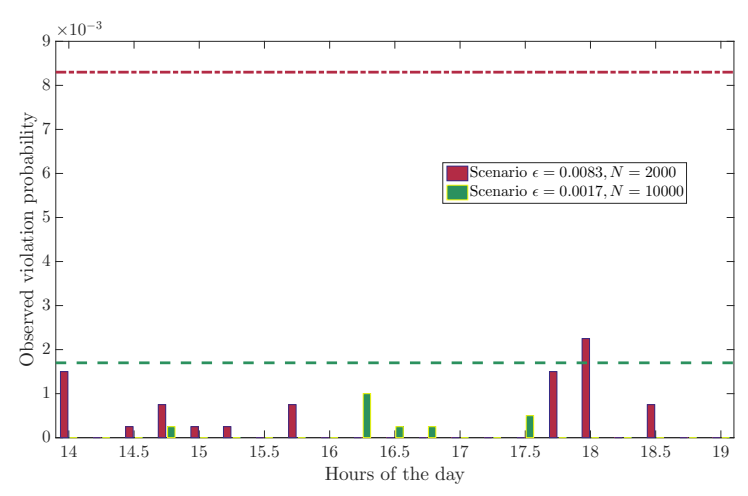

Fig. 4: $V\left(x_{\mathcal{S}}^{\star}\right)$ for two different scenario settings.

\section{Case Study}

In this section, we test the proposed approach on a 2000bus synthetic grid on a footprint of Texas [54]. This system consists of 544 generation units, with a portfolio of 367 gas, 39 coal, 4 nuclear, 25 hydro, 87 wind and 22 utility scale solar power plants. Nodes with wind/solar resources are where uncertainty exists. This can be generalized to DER aggregation and participation into the wholesale electricity market. 432 of these units are active during the study period (default setting in [54]). Its transmission network consist of 3206 transmission lines. Installed wind capacity is about $13 \%$ of the peak load, and installed solar capacity is less than $1 \%$ of the net load. MATPOWER [55] is used to obtain PTDF of the synthetic grid and confirm the accuracy of the base case modelings. Where data was not given (such as the ramping capabilities of the units), the modifications were performed according to [10], [39]. In addition load and wind profiles were adapted from these references.

The optimization is performed for a 24 hour period ( 96 intervals). $T$ in (5) is two, meaning that there is one deterministic and binding, and one uncertain, non-binding interval. For efficient illustration, in each of the following subsections, the focus will be on some different windows of the 96 intervals during a day. It is assumed that generators bid linearly into the real-time market. The uncertainty on each uncertain resource is distributed according to Gaussian distribution with mean $\mu$ equal to the nominal forecast and with standard deviation $\sigma$ 

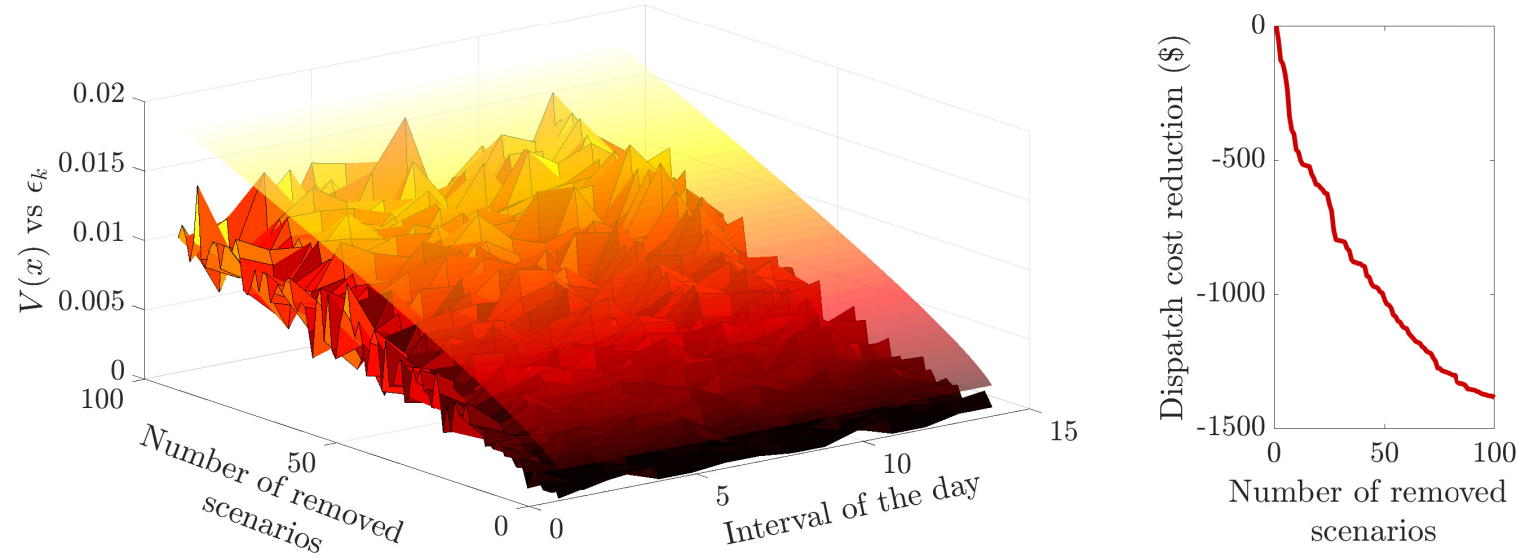

Fig. 5: Sampling and discarding results: trading risk for performance. Left: Violation probability (Monte Carlo estimate with 10000 samples) located below $\epsilon_{k}$. Right: Binding interval cost reduction in one interval after elimination of $k$ scenarios.

defined as the normalized standard deviation of the wind/solar forecast. A scenario is obtained by sampling the uncertainty instances from these distributions in an independent fashion. Information on the scenario generation mechanism was provided here for the sake of comparison only, and it must be remarked that the adopted method does not require that the underlying probability distribution be known. Deviations from forecasted values enter the net load scenarios as negative load. The confidence parameter $\beta=10^{-6}$ is used throughout the case study. The decision of each dispatch method is tested using 10000 independent scenarios extracted from the same uncertainty set.

This case study is divided into two parts. The focus of the first part is on the ramping events due to renewable integration in the system, illustrating the algorithm suggested in Subsections III-A and III-B with $d=1$ in the absence of congestion in the system. The second part extends the original scenario theory to the results shown in Subsection III-C in the presence of line constraints. It is shown that by using the results in (12) it is possible to start with a sample size with almost no guarantee on the results and reach a very high confidence in the results by analyzing the complexity of the solution.

\section{A. Extreme ramping test: Scenario vs deterministic and robust $L A E D$}

To simulate how different methods respond to the possibility of an extreme ramping event, we increased the wind/solar penetration threefold while increasing the load in the system by $18 \%$. $\sigma$ for each uncertain resource is $0.07 \mu$, where we recall that $\mu$ is the forecast of wind and solar resources. A full Gaussian distribution is used to generate the scenarios for the scenario approach. Following the robust methodology in [17], we truncated the Gaussian distribution at $\mu \pm 3 \sigma$ for the robust method.

The simulation is performed for two different sizes of scenarios, and compared to the deterministic and robust methods. The scenario sizes are 2000 and 10000 , which correspond to $\epsilon=0.0083$ and 0.0017 respectively using (8). As discussed in
Section II, the decision for the first interval is binding and the future interval is advisory. Therefore in Fig. 3, we compare the dispatch cost of the binding interval (where there is no uncertainty) using different approaches. We show peak hours in Fig. 3 because the system is more vulnerable to ramping events during these hours. As can be seen, the robust method has a clear offset in terms of the binding dispatch cost while the deterministic method carries the least cost of dispatch. However, the increment in the dispatch cost using the scenario method is small compared to the robust method. It should be noted that the generated sets of 2000 and 10000 scenarios are generated independently. Therefore there can be a few cases where the dispatch cost is higher with 2000 scenarios than with 10000.

Violation probabilities in the scenario approach are as expected and shown in Fig. 4. The robust method maintained the zero violation probability, while scenario LAED allowed some violations, but kept this violation below the corresponding $\epsilon$. The $V\left(x_{\mathcal{S}}^{\star}\right)$ for the deterministic LAED is 0.5029 for the hours shown in Fig. 4. Therefore, the scenario method successfully confines $V\left(x_{\mathcal{S}}^{\star}\right) \leq \epsilon$ with a cost much smaller than the robust method.

Some extreme scenarios that can lead to conservative results might be included when samples are being collected randomly. We used 10000 scenarios in the previous section and dropped up to 100 of them. As mentioned in Section III-B, the discarding strategy can be using any arbitrary rule. In this case, we discard the constraints whose removal maximizes the reduction of dispatch cost. As shown in Fig. 5 (right), when scenarios are being dropped, the performance, which in this case is the cost of the binding interval, is being improved. The performance improvement is traded for risk. Fig. 5 (left) shows $V\left(x_{\mathcal{S}-k}^{\star}\right)$ and $\epsilon_{k}$ after dropping $k \in[1,100]$ scenarios. The values of $\epsilon_{k}$ extracted from (10) are the values of the transparent plane depicted above the observed violation probabilities.

Trading risk for performance can be particularly helpful if dropping the first few scenarios significantly reduces the costs, as in the case of the first few scenarios in Fig. 5 (right). 


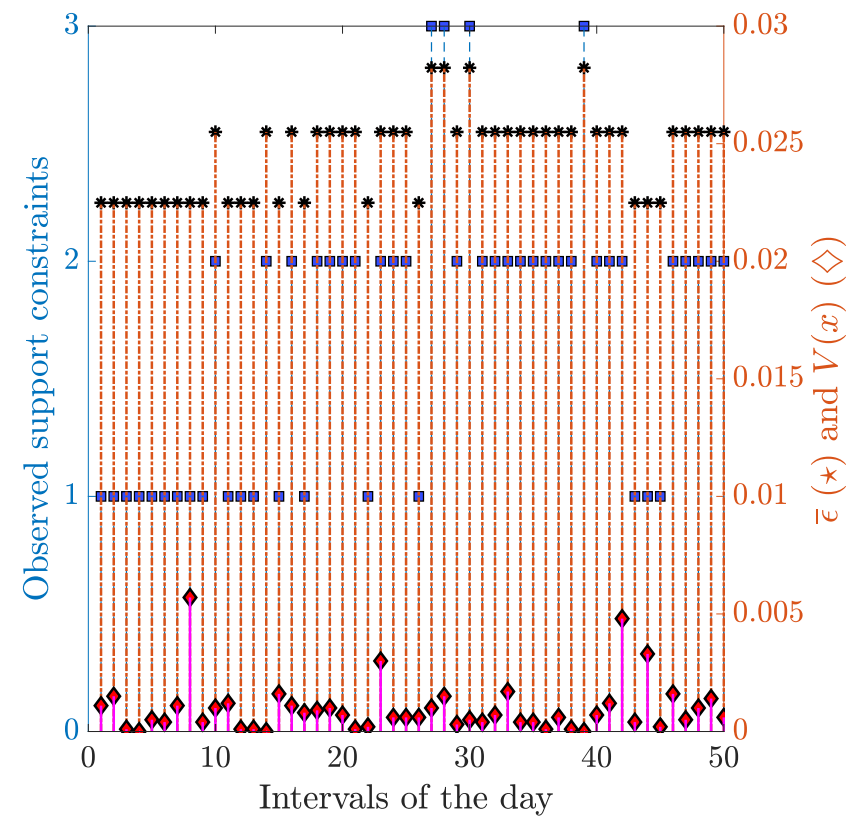

Fig. 6: $\square$ : Number of observed support constraints, $\diamond$ : violation probability (Monte Carlo estimate with 10000 samples) and $\star$, the upper bound on the violation probability based upon the complexity.

\section{B. Risk and complexity: Considering all constraints in the Sc- $L A E D$}

In this subsection, both network and ramping constraints are considered. Therefore it is no longer possible to know the exact number of support constraints prior to solving the problem. To be able to use the original line constraints in [54], we do not change wind and solar penetration in this section. However, to cause congestion, we changed the load by $5 \%$ at all nodes. The argument is that by making a guess that the number of support constraints is low, we can start with a very large $\epsilon$, solve the problem, and by observing the results update our knowledge of $\epsilon$. In this case we solved the problem with 870 scenarios, which is slightly more than the number of decision variables (which is 864). This leads to $\epsilon=0.9996$. This means that $V\left(x_{\mathcal{S}}^{\star}\right)$ can vary from 0 to 0.9996 , so that Theorem 1 provides almost no information about $V\left(x_{\mathcal{S}}^{\star}\right)$. However, an a-posteriori upper bound for $V\left(x_{\mathcal{S}}^{\star}\right)$ can be found by Theorem. 2 .

For instance, when 3 constraints of support are observed in Sc-LAED, meaning that their removal changes the solution, the claim " $0 \leq V\left(x_{\mathcal{S}}^{\star}\right) \leq 0.0282$ " can be delivered.

For the test case, a-posteriori results for the first 50 intervals of a day are summarized in Fig. 6. As can be seen, the observed number of support constraints (blue $\square$ ) is small, although congestion exists. The number of support constraints for this study varies between one, two and, for some intervals three, which is much smaller than $d=864$ (while the apriori results in [28] are for a fully supported problem, i.e., $\nu_{\mathcal{S}}^{\star}=864$ with probability one). Using Theorem 2, one can rigorously define an upper bound on the risk of dispatch for these intervals. Our knowledge about the upper level of $V(x)$ gets much sharper as shown by the black stars in Fig. 6 (compare $V(x) \leq 0.9996$ with the results). 10000 samples for each interval were used to estimate the violation probability: the resulting estimates are all within the theoretical bounds and are represented by the red $\diamond$ in Fig. 6 .

\section{CONClusion}

In this paper, the scenario approach for solving uncertain economic dispatch is introduced. It is shown that this approach does not require any knowledge of the underlying uncertainty distribution, yet yields a quantifiable level of risk in real-time economic dispatch. It is shown how the risk can be evaluated according to a-priori and a-posteriori mathematical results. Scalability of the problem is considered in both the a-priori and a-posteriori stages.

In the a-priori stage, it is shown that disregarding congestion, the number of samples needed does not increase with the size of the system. This fact bears several benefits: first, it makes the process of collecting i.i.d. samples practical; second, it avoids both an overly conservative solution as well as high computational burden. Moreover, pessimistic scenarios can be neglected with controllable degradation of the violation probability.

In the a-posteriori stage, the risk of constraint violation can turn out to be much smaller than general a-priori, promising future scalability of the Sc-LAED for a congested case. The case study on a realistic power system suggests that scenario based LAED could provide a reliable solution with quantifiable bound on the conservativeness of the results.

There is a need for more rigorous investigations of the correlation between the number of constraints of support and design parameters in Sc-LAED. Therefore, our future work will be mainly focused on the a-posteriori stage, where a procedure to start from a few scenarios and progressively aim towards desired $\bar{\epsilon}$ based on the observed number of support constraints, will be developed.

Practically speaking, the scenario approach strikes a good trade-off between deterministic and robust optimization-based dispatch. The ISO could potentially adopt scenario approach as a natural step to manage uncertain DERs while keeping a tunable risk level at the ex-post stage. It could have direct benefits to both real-time and intra-day decision making process.

\section{ACKNOWLEDGEMENT}

Portions of this research were conducted with the advanced computing resources provided by Texas A\&M High Performance Research Computing. 
TABLE II: Appendix Nomenclature

Sets:

$\mathbf{C L}^{+} / \mathbf{C L}^{-} \quad$ Set of positively/negatively congested lines. Parameters and constants:

\begin{tabular}{|c|c|}
\hline$\hat{P}_{g_{i}}$ & $\begin{array}{l}\text { Decision input from binding interval of Sc- } \\
\text { LAED. }\end{array}$ \\
\hline$\rho$ & Energy balance equation shadow price. \\
\hline$\varrho_{\max }$ & $\begin{array}{l}\text { Shadow price corresponded to a positively } \\
\text { congested lines. }\end{array}$ \\
\hline$\varrho_{\min }$ & $\begin{array}{l}\text { Shadow price corresponded to a negatively } \\
\text { congested lines. }\end{array}$ \\
\hline$\varsigma$ & $\begin{array}{l}\text { Shadow price for generation capacity con- } \\
\text { straint. }\end{array}$ \\
\hline & bles: \\
\hline & Power generation for $g_{i}$. \\
\hline
\end{tabular}

\section{APPENDIX A}

SC-LAED Locational MARginal Pricing

$$
\begin{array}{ll}
\min _{P_{g_{i}}[t]} & z=\sum_{i=1}^{N_{g}} c_{g_{i}} P_{g_{i}} \\
\text { s.t. } & \\
\rho & \sum_{i=1}^{N_{g}} P_{g_{i}}=\sum_{i=1}^{N_{g}} \hat{P}_{g_{i}} \\
& \sum_{\text {max }}^{N_{b}} P T D F_{\mathbf{e}}^{\varpi^{+}} \mathcal{P}_{\mathbf{n}}^{\varpi^{+}} \leq \overline{F^{\varpi^{+}}}, \quad \forall \varpi^{+} \in \mathbf{C L}^{+},(13 \mathrm{c} \\
& \varrho_{\min } \quad \sum_{j=1}^{N_{b}} P T D F_{\mathbf{e}}^{\varpi^{-}} \mathcal{P}_{\mathbf{n}}^{\varpi^{-}} \geq-\overline{F^{-}}, \quad \forall \varpi^{-} \in \mathbf{C L}^{-}, \\
& \hat{P}_{g_{i}}^{\min } \leq P_{g_{i}} \leq \hat{P}_{g_{i}}^{\max } \quad \forall i=1,2, \ldots, N_{g} .
\end{array}
$$

The presented LMP calculation process is based on equations (1) to (5) in [45]. For simplicity, here we have assumed loss-less system. In the following optimization problem, a hat symbol represents the input to the problem from the Sc-LAED. The objective is to minimize total generation cost (13a). (13b) is energy balancing constraint to satisfy the same demand as (5b). For a set of positively and negatively congested lines in (13c) and (13d) return shadow prices corresponding to the line constraints. Capacity constraint of each generator embedded with its incremental ramping up $\Delta P_{g_{i}}^{\max }$ and down $\Delta P_{g_{i}}^{\min }$ limit of such generator is shown in (13e), where $\hat{P}_{g_{i}}^{g_{i} a x}=\hat{P}_{g_{i}}+\Delta P_{g_{i}}^{\max }$ and $\hat{P}_{g_{i}}^{\text {min }}=\hat{P}_{g_{i}}+\Delta P_{g_{i}}^{\min }$.

The $N_{b} \times 1$ vector of nodal LMP can be reached by (14). $\mathbf{1}_{N_{b}}$ is a $N_{b} \times 1$ all ones column vector, and $\varrho_{\kappa}^{\max }$ and $\varrho_{\kappa}^{\min }$ are $N_{l} \times 1$ column matrices containing $\varrho^{\max }$ and $\varrho^{\min }$ on the rows corresponding to a positively or negatively congested line respectively.

$$
\mathbf{L M P}=\rho(\kappa) \times \mathbf{1}_{N_{b}}-P T D F_{\mathbf{e}}^{\prime} \times\left(\varrho_{\kappa}^{\max }-\varrho_{\kappa}^{\min }\right)
$$

\section{REFERENCES}

[1] "ERCOT quick facts," (Date last accessed 30-September-2018). [Online]. Available: https://goo.gl/x7cCXc
[2] “2016 LTSA update,” (Date last accessed 30-September-2018). [Online]. Available: https://goo.gl/9fo9FD

[3] P. P. Varaiya, F. F. Wu, and J. W. Bialek, "Smart Operation of Smart Grid: Risk-Limiting Dispatch," Proceedings of the IEEE, vol. 99, no. 1, pp. 40-57, 2011.

[4] R. Entriken, P. Varaiya, F. Wu, J. Bialek, C. Dent, A. Tuohy, and R. Rajagopal, "Risk limiting dispatch," in 2012 IEEE Power and Energy Society General Meeting, July 2012, pp. 1-5.

[5] L. Wu, M. Shahidehpour, and T. Li, "Stochastic Security-Constrained Unit Commitment," IEEE Transactions on Power Systems, vol. 22, no. 2, pp. 800-811, 2007.

[6] A. Papavasiliou, S. S. Oren, and R. P. O. Neill, "Reserve Requirements for Wind Power Integration: A Stochastic Programming Framework," IEEE Transactions on Power Systems, vol. 26, no. 4, pp. 2197-2206, 2011.

[7] D. Bertsimas, E. Litvinov, X. A. Sun, J. Zhao, and T. Zheng, "Adaptive Robust Optimization for the Security Constrained Unit Commitment Problem," IEEE Transactions on Power Systems, vol. 28, no. 1, pp. 52-63, 2013.

[8] N. Zhang, C. Kang, Q. Xia, Y. Ding, Y. Huang, R. Sun, J. Huang, and J. Bai, "A convex model of risk-based unit commitment for day-ahead market clearing considering wind power uncertainty," IEEE Transactions on Power Systems, vol. 30, no. 3, pp. 1582-1592, May 2015.

[9] D. Ross and S. Kim, "Dynamic Economic Dispatch of Generation," IEEE Transactions on Power Apparatus and Systems, vol. PAS-99, no. 6, pp. 2060-2068, 1980.

[10] Y. Gu and L. Xie, "Early detection and optimal corrective measures of power system insecurity in enhanced look-ahead dispatch," IEEE Transactions on Power Systems, vol. 28, no. 2, pp. 1297-1307, 2013.

[11] Z. Li, W. Wu, B. Zhang, and H. Sun, "Efficient Location of Unsatisfiable Transmission Constraints in Look-Ahead Dispatch via an Enhanced Lagrangian Relaxation Framework," vol. 30, no. 3, pp. 1-10, 2014.

[12] Á. Lorca and X. A. Sun, "Adaptive Robust Optimization With Dynamic Uncertainty Sets for Multi-Period Economic Dispatch," IEEE Transactions on Power Systems, vol. 30, no. 4, pp. 1702-1713, 2015.

[13] Q. Wang and B. M. Hodge, "Enhancing power system operational flexibility with flexible ramping products: A review," IEEE Transactions on Industrial Informatics, vol. 13, no. 4, pp. 1652-1664, Aug 2017.

[14] H. Nosair and F. Bouffard, "Economic dispatch under uncertainty: The probabilistic envelopes approach," IEEE Transactions on Power Systems, vol. 32, no. 3, pp. 1701-1710, May 2017.

[15] C. Tang, J. Xu, Y. Sun, J. Liu, X. LI, D. Ke, J. Yang, and X. Peng, "Look-ahead economic dispatch with adjustable confidence interval based on a truncated versatile distribution model for wind power," IEEE Transactions on Power Systems, vol. PP, no. 99, pp. 1-1, 2017.

[16] Y. Gu and L. Xie, "Stochastic Look-Ahead Economic Dispatch With Variable Generation Resources," IEEE Transactions on Power Systems, pp. 1-13, 2016.

[17] A. A. Thatte and L. Xie, "A metric and market construct of intertemporal flexibility in time-coupled economic dispatch," IEEE Transactions on Power Systems, vol. 31, no. 5, pp. 3437-3446, 2016.

[18] A. A. Thatte, X. A. Sun, and L. Xie, "Robust optimization based economic dispatch for managing system ramp requirement," Proceedings of the Annual Hawaii International Conference on System Sciences, pp. 2344-2352, 2014.

[19] A. Ben-Tal and A. Nemirovski, "Robust convex optimization," Mathematics of operations research, vol. 23, no. 4, pp. 769-805, 1998.

[20] L. El Ghaoui, F. Oustry, and H. Lebret, "Robust solutions to uncertain semidefinite programs," SIAM Journal on Optimization, vol. 9, no. 1, pp. 33-52, 1998.

[21] A. Ben-Tal and A. Nemirovski, "On tractable approximations of uncertain linear matrix inequalities affected by interval uncertainty," SIAM Journal on Optimization, vol. 12, no. 3, pp. 811-833, 2002.

[22] G. Calafiore and M. C. Campi, "The scenario approach to robust control design," IEEE Transactions on Automatic Control, vol. 51, no. 5, pp. 742-753, 2006.

[23] A. Prékopa, Stochastic programming. Springer Science \& Business Media, 2013.

[24] M. W. Tanner and L. Ntaimo, "IIS branch-and-cut for joint chanceconstrained stochastic programs and application to optimal vaccine allocation," European Journal of Operational Research, vol. 207, no. 1, pp. $290-296,2010$.

[25] B. Zeng, Y. An, and L. Kuznia, "Chance constrained mixed integer program: Bilinear and linear formulations, and benders decomposition," Mathematical Programming (to appear), 2017. 
[26] A. Nemirovski and A. Shapiro, "Convex approximations of chance constrained programs," SIAM Journal on Optimization, vol. 17, no. 4, pp. 969-996, 2006.

[27] A. Ben-Tal, L. El Ghaoui, and A. Nemirovski, Robust optimization. Princeton University Press, 2009.

[28] M. C. Campi and S. Garatti, "The Exact Feasibility of Randomized Solutions of Uncertain Convex Programs," SIAM Journal on Optimization, vol. 19, no. 3, pp. 1211-1230, 2008.

[29] —, "A sampling-and-discarding approach to chance-constrained optimization: feasibility and optimality," Journal of Optimization Theory and Applications, vol. 148, no. 2, pp. 257-280, 2011.

[30] K. Margellos, P. Goulart, and J. Lygeros, "On the road between robust optimization and the scenario approach for chance constrained optimization problems," IEEE Transactions on Automatic Control, vol. 59, no. 8 , pp. 2258-2263, Aug 2014.

[31] S. Grammatico, X. Zhang, K. Margellos, P. Goulart, and J. Lygeros, "A scenario approach to non-convex control design: Preliminary probabilistic guarantees," in 2014 American Control Conference, June 2014, pp. 3431-3436.

[32] M. Vrakopoulou, K. Margellos, J. Lygeros, and G. Andersson, "A probabilistic framework for reserve scheduling and $\mathrm{N}-1$ security assessment of systems with high wind power penetration," IEEE Transactions on Power Systems, vol. 28, no. 4, pp. 3885-3896, 2013.

[33] Y. Zhang, S. Shen, and J. L. Mathieu, "Distributionally robust chanceconstrained optimal power flow with uncertain renewables and uncertain reserves provided by loads," IEEE Transactions on Power Systems, vol. 32, no. 2, pp. 1378-1388, March 2017.

[34] M. Vrakopoulou, B. Li, and J. L. Mathieu, "Chance constrained reserve scheduling using uncertain controllable loads part I: Formulation and scenario-based analysis," IEEE Transactions on Smart Grid, 2017.

[35] H. Ming, L. Xie, M. C. Campi, S. Garatti, and P. R. Kumar, "Scenariobased economic dispatch with uncertain demand response," IEEE Transactions on Smart Grid (accepted, to appear), 2017.

[36] M. C. Campi and S. Garatti, "Wait-and-judge scenario optimization," Mathematical Programming, vol. 167, no. 1, pp. 155-189, 2018.

[37] A. J. Wood and B. F. Wollenberg, Power generation, operation, and control. John Wiley \& Sons, 2012.

[38] G. Calafiore and M. C. Campi, "Uncertain convex programs: randomized solutions and confidence levels," Mathematical Programming, vol. 102, no. 1, pp. 25-46, 2005.

[39] A. A. Thatte, Y. Li, and L. Xie, "Managing system ramp flexibility by utilizing price-responsive demand: An empirical assessment," in 2016 49th Hawaii International Conference on System Sciences (HICSS). IEEE, 2016, pp. 2345-2353.

[40] H. A. Nasir, T. Zhao, A. Carè, Q. J. Wang, and E. Weyer, "Efficient river management using stochastic MPC and ensemble forecast of uncertain in-flows," in IFAC-PapersOnLine. Elsevier, 2018, pp. 37-42.

[41] S. Garatti and M. C. Campi, "Modulating robustness in control design: Principles and algorithms," IEEE Control Systems, vol. 33, no. 2, pp. 36-51, April 2013.

[42] T. Zheng and E. Litvinov, "On ex post pricing in the real-time electricity market," IEEE Transactions on Power Systems, vol. 26, no. 1, pp. 153164, 2011.

[43] - "Ex post pricing in the co-optimized energy and reserve market," IEEE Transactions on Power Systems, vol. 21, no. 4, pp. 1528-1538, 2006.

[44] A. L. Ott, "Experience with PJM market operation, system design, and implementation," IEEE Transactions on Power Systems, vol. 18, no. 2 , pp. 528-534, 2003.

[45] F. Li, Y. Wei, and S. Adhikari, "Improving an unjustified common practice in ex post LMP calculation," IEEE Transactions on Power Systems, vol. 25, no. 2, pp. 1195-1197, 2010.

[46] D. H. Choi and L. Xie, "Economic impact assessment of topology data attacks with virtual bids," IEEE Transactions on Smart Grid, vol. 9, no. 2, pp. 512-520, March 2018

[47] W. W. Hogan et al., "Financial transmission rights, revenue adequacy and multi-settlement electricity markets," unpublished.[Online]. Available Harvard University web site: http://www. hks. harvard. edu/fs/whogan/Hogan_FTR_Rev_Adequacy_, vol. 31813, 2012.

[48] M. C. Campi, S. Garatti, and M. Prandini, "The scenario approach for systems and control design," IFAC Proceedings Volumes, vol. 41, no. 2 , pp. 381-389, 2008.

[49] G. Schildbach, L. Fagiano, and M. Morari, "Randomized solutions to convex programs with multiple chance constraints," SIAM Journal on Optimization, vol. 23, no. 4, pp. 2479-2501, 2013.
[50] X. Zhang, S. Grammatico, G. Schildbach, P. Goulart, and J. Lygeros, "On the sample size of random convex programs with structured dependence on the uncertainty," Automatica, vol. 60, pp. 182-188, 2015.

[51] A. Carè, S. Garatti, and M. C. Campi, "Fast-fast algorithm for the scenario technique," Operations Research, vol. 62, no. 3, pp. 662-671, 2014.

[52] M. C. Campi and A. Carè, "Random convex programs with $L_{1}$ regularization: Sparsity and generalization," SIAM Journal on Control and Optimization, vol. 51, no. 5, pp. 3532-3557, 2013.

[53] V. L. Levin, "Application of E. Helly's theorem to convex programming, problems of best approximation and related questions," Sbornik: Mathematics, vol. 8, no. 2, pp. 235-247, 1969.

[54] A. B. Birchfield, K. M. Gegner, T. Xu, K. S. Shetye, and T. J. Overbye, "Statistical considerations in the creation of realistic synthetic power grids for geomagnetic disturbance studies," IEEE Transactions on Power Systems, vol. 32, no. 2, pp. 1502-1510, March 2017.

[55] R. D. Zimmerman, C. E. Murillo-Sanchez, and R. J. Thomas, "MATPOWER: Steady-state operations, planning, and analysis tools for power systems research and education," IEEE Transactions on Power Systems, vol. 26, no. 1, pp. 12-19, Feb 2011.

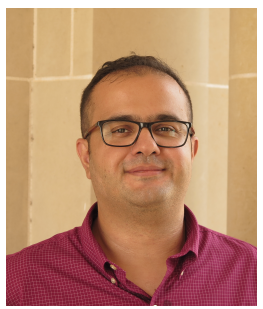

Mohammad Sadegh Modarresi (Sadegh) (S'13) received his B.Sc. degree from Iran University of Science and Technology (IUST), Tehran, Iran, and M.Sc. degree from Sharif University of Technology, Tehran, Iran, in electrical engineering in 2008 and 2011 respectively. He is currently a research assistant and Ph.D. student at the Department of Electrical and Computer Engineering at Texas A\&M University, College Station, TX, USA. His research interests include energy and ancillary service markets, demand participation in bulk power systems, and application of big data analytics in distribution system operations.

His industry experiences include internships at National Rural Electric Cooperative Association (NRECA), Market analysis group of Electric Reliability Council of Texas (ERCOT) and transmission and distribution operations at Dominion Energy in 2015, 2016, and 2018 respectively.

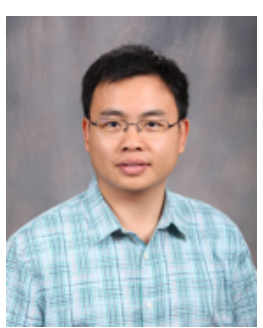

Le Xie (S'05-M'10-SM'16) received the B.E. degree in electrical engineering from Tsinghua University, Beijing, China, in 2004, the M.S. degree in engineering sciences from Harvard University, Cambridge, MA, USA, in 2005, and the Ph.D. degree from the Department of Electrical and Computer Engineering, Carnegie Mellon University, Pittsburgh, PA, USA in 2009. He is currently a Professor with the Department of Electrical and Computer Engineering, Texas A\&M University, College Station, TX, USA. His research interests include modeling and control of large-scale complex systems, smart grids application with renewable energy resources, and electricity markets.

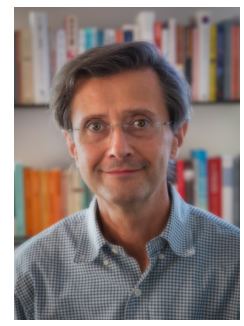

Marco Claudio Campi is professor of control and inductive methods at the University of Brescia, Italy. $\mathrm{He}$ has been in various capacities on the Editorial Board of Automatica, Systems \& Control Letters and the European Journal of Control, and he was, until 2017, the chair of the Technical Committee IFAC on Modeling, Identification and Signal Processing (MISP). Marco Campi is a recipient of the "Giorgio Quazza" prize, and he received in 2008 the IEEE CSS George S. Axelby outstanding award for the article "The Scenario Approach to Robust Control Design". He has delivered plenary and semi-plenary addresses at major conferences including CDC, SYSID, ECC, and MTNS. Currently he is a distinguished lecturer of the Control Systems Society. Marco Campi is a Fellow of IEEE, a member of IFAC, and a member of SIDRA. 


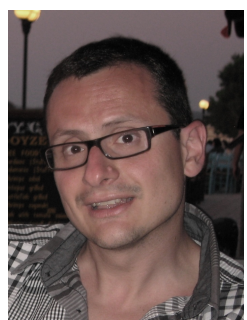

Simone Garatti is Associate Professor at the Dipartimento di Elettronica ed Informazione of the Politecnico di Milano, Milan, Italy. He received the Laurea degree and the Ph.D. in Information Technology Engineering in 2000 and 2004, respectively, both from the Politecnico di Milano. In 2003, he was visiting scholar at the Lund University of Technology, Lund, Sweden, in 2006 at the University of California San Diego (UCSD), San Diego, CA, USA, and in 2007 at the Massachusetts Institute of Technology and the Northeastern University, Boston, MA, USA. He is member of the IEEE Technical Committee on Computational Aspects of Control System Design and of the IFAC Technical Committee on Modeling, Identification and Signal Processing. His research interests include data-based and stochastic optimization for problems in systems and control, system identification, model quality assessment, and uncertainty quantification.

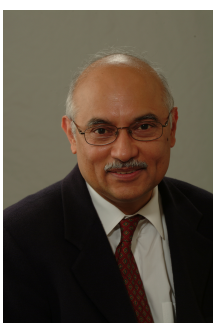

P. R. Kumar B. Tech. (IIT Madras, '73), D.Sc. (Washington University, St. Louis, '77), was a faculty member at UMBC (1977-84) and Univ. of Illinois, Urbana-Champaign (1985-2011). He is currently at Texas A\&M University. His current research is focused on cyber-physical systems, cybersecurity, privacy, wireless networks, renewable energy, smart grid, autonomous vehicles, and unmanned air vehicle systems.

$\mathrm{He}$ is a member of the US National Academy of Engineering, The World Academy of Sciences, and the Indian National Academy of Engineering. He was awarded a Doctor Honoris Causa by ETH, Zurich. He has received the IEEE Field Award for Control Systems, the Donald P. Eckman Award of the AACC, Fred W. Ellersick Prize of the IEEE Communications Society, the Outstanding Contribution Award of ACM SIGMOBILE, the Infocom Achievement Award, and the SIGMOBILE Test-of-Time Paper Award. He is a Fellow of IEEE and ACM Fellow. He was Leader of the Guest Chair Professor Group on Wireless Communication and Networking at Tsinghua University, is a D. J. Gandhi Distinguished Visiting Professor at IIT Bombay, and an Honorary Professor at IIT Hyderabad. He was awarded the Distinguished Alumnus Award from IIT Madras, the Alumni Achievement Award from Washington Univ., and the Daniel Drucker Eminent Faculty Award from the College of Engineering at the Univ. of Illinois.

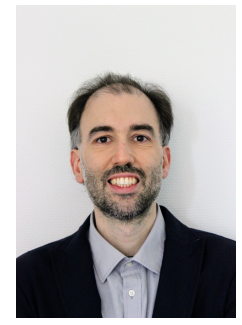

Algo Carè received the Ph.D. degree in informatics and automation engineering from the University of Brescia, Brescia, Italy, in 2013. He is currently a Research Fellow with the Department of Information Engineering, University of Brescia. He spent two years at The University of Melbourne, Melbourne, VIC, Australia, as a Research Fellow in system identification with the Department of Electrical and Electronic Engineering. Dr. Carè was a recipient of a two-year ERCIM Fellowship in 2016 that he spent at the Institute for Computer Science and Control (SZTAKI), Hungarian Academy of Sciences (MTA), Budapest, Hungary, and at the Multiscale Dynamics Group, National Research Institute for Mathematics and Computer Science (CWI), Amsterdam, The Netherlands. He received the triennial Stochastic Programming Student Paper Prize by the Stochastic Programming Society for the period 2013-2016. His current research interests include data-driven decision methods, system identification, and learning theory.

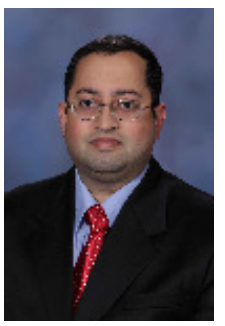

Anupam Thatte received his B.E. in Electrical Engineering from Pune University, India; an M.S. in Electrical and Computer Engineering from Carnegie Mellon University; and a Ph.D. in Electrical and Computer Engineering from Texas A\&M University.

$\mathrm{He}$ is currently working for the Midcontinent Independent System Operator. His research interests include modeling and control of power systems, grid integration of renewable energy and electricity markets. 